\title{
A study on COVID-19 transmission dynamics: Stability analysis of SEIR model with Hopf bifurcation for effect of time delay
}

\author{
M.Rasha \\ SRM IST
}

S.Balamuralitharan ( $\square$ balamurs@srmist.edu.in )

SRM IST

\section{Research Article}

Keywords: Covid19, SEIR, Stability, Hopf bifurcation.

Posted Date: May 7th, 2020

DOI: https://doi.org/10.21203/rs.3.rs-27137/v1

License: (c) (1) This work is licensed under a Creative Commons Attribution 4.0 International License.

Read Full License 


\title{
A study on COVID-19 transmission dynamics: Stability analysis of SEIR model with Hopf bifurcation for effect of time delay
}

\author{
M.Radha ${ }^{1}$, S.Balamuralitharan ${ }^{2}$ \\ ${ }^{1,2}$ Faculty of Engineering and Technology, Department of Mathematics, \\ SRM Institute of Science and Technology, Kattankulathur - 603 203, Tamil Nadu, INDIA. \\ ${ }^{2}$ Email : balamurs@srmist.edu.in
}

\begin{abstract}
This paper deals with a general SEIR model for the coronavirus disease 2019 (COVID-19) with the effect of time delay proposed. We get the stability theorems for disease-free equilibrium and provide adequate situations of the COVID-19 transmission dynamics equilibrium of present and absent cases. A Hopf bifurcation parameter $\tau$ is the effects of time delay and we demonstrate that the locally asymptotic stable is present equilibrium. The Reproduction number is brief in less than or greater than one, and it effectively controlling the COVID-19 infection outbreak, and subsequently reveals insight into understanding the patterns of the flare-up. The numerical experiment is calculated to help the theoretical outcomes.
\end{abstract}

Keywords: Covid19, SEIR, Stability, Hopf bifurcation.

AMS Subject Classification: 34D20, 34E05, 34K50.

\section{Introduction}

As of 18 April 2020 (5:00 PM), as per the Ministry of Health and Family Welfare (MoHFW), India, an aggregate of 14792 COVID-19 cases, (counting 76 outside nationals) have been accounted for in 32 sta[1]. These incorporate 2014 who have been released, 1 who has relocated and 488 deaths [2]. Medical clinic segregation of every single confirmed case, tracing and home quarantine of the contacts is ongoing (Globally: 2074529 confirmed, 139378 deaths). The progressing COVID-19 episode, developed in Wuhan, China, has guaranteed more than 2600 lives starting on 24 February 2020 and represented a colossal danger to worldwide general wellbeing [3]-[8]. It has actualized different methods adding current situations uncommon medical clinics and travel limitations to relieve the infection. Here we have indicated that coronavirus ailment has been engendered in the network of China quickly by revealed information and the Government of China make important strides, for example, occasion augmentation, travel limitation, hospitalization and isolate. These vital limitations have been useful to diminish the infection transmission among the populace and this is legitimized by information results [9]-[15]. It is earnest to give increasingly logical data to a superior comprehension of the noval coronavirus and promote control of the outbreak [16].

At the beginning of time episode, was dispersed, and connected to the Market places [17]. It has received outrageous measures to relieve flare-up. On 10 March 2020, the neighborhood legislature of Wuhan controlled every open traffic inside the city and shut all inbound and outbound transportation [18]-[24]. The open frenzy progressing COVID-19 episode helps us the records to remember the 1920 flu pandemic in London, United Kingdom. Besides, its attributes of mellow side effects as a rule and short sequential interim are like pandemic flu, as opposed to the next two coronaviruses [25]. In 1918, critical extents of the passing were from pneumonia followed flu contamination [26]. Hence, it may be sensible to return to the demonstrating system of 1918 flu pandemic, and specifically, to catch the impacts of the individual response and government activity. We assume it will keep going for the following not many days for the occasion and shall refresh. The variables esteem might be evaluated after that data is accessible [27]-[30]. It contends all avoidance and limiting cases might be ordered up to three huge gatherings, that are portrayed a stage work and reaction work, separately. They likewise consider COVID-19 transmission time of 14 days and gigantic resettlement from China [31]. A contact is an individual who encountered any of the accompanying 
exposures during the 2 days prior and the 14 days after the beginning of side effects of a plausible or affirmed case [32].

For transmission of COVID-19 among the human population and its stability we have a proposed SEIR pandemic model [33]. Another scientific model in pestilence elements, known as the Warehouse theory, generally has been discussed for quite a while since it was sent by Kermack and McKendrick in 1927. It incorporates a few essential improved models, for example, SIR, SIS, SEIR, etc, among which SEIR is an ordinary model that considers the incubation period into account. The SEIR, a widely utilized scourge model, can show the progressions of individuals between four states: Susceptible (S) (Population not resistant to illness), Exposed (E) (Population as of now in brooding), Infectious (I) (Number of contamination effectively circling), and Recovered (R) (Population not, at this point irresistible because of confinement or in susceptibility or Full recuperation). Here the population total size at time $t$ is defined by $\mathrm{N}(\mathrm{t})$, with $\mathrm{N}(\mathrm{t})=\mathrm{S}(\mathrm{t})+\mathrm{E}(\mathrm{t})+\mathrm{I}(\mathrm{t})+\mathrm{R}(\mathrm{t})$. This system is portrayed by the accompanying nonlinear differential equations [34]:

$$
\left\{\begin{array}{l}
\frac{d S(t)}{d t}=b+\gamma I(t)-(\mu+p) S(t)-\beta S(t) I(t) \\
\frac{d E(t)}{d t}=\beta S(t) I(t)-\mu E(t)-\eta E(t) R(t) \\
\frac{d I(t)}{d t}=(\eta+\sigma \beta) E(t)-(\alpha+\mu+\gamma) I(t) \\
\frac{d R(t)}{d t}=p S(t) E(t) R(t)-(\mu+\sigma \beta) R(t)
\end{array}\right.
$$

The parameters $\mathrm{p}, \mathrm{b}, \gamma, \beta, \mu, \eta, \sigma, \alpha$ are positive constants, $\mathrm{p}$ is the proportion of asymptomatic infection, $\mathrm{b}$ is the birth rate of people while newborn cells are created, $\gamma$ is the incubation period of human infection, $\beta$ is the transmission rate from one compartment to another compartment, $\mu$ is the death rate of people, $\eta$ is the infectious period of symptomatic infection of people, $\sigma$ is the infectious period of asymptomatic infection of people, $\alpha$ is the multiple of the transmissibility while infected cells are created from the viruses.

Transmission dynamics generating COVID-19 may require a duration of time delay $\tau$, i.e. the delay of the immune system at time (days) $t$ may be governed on the previous time $t-\tau$. We obtain an immune response of length of incubation period, $p S(t) E(t) R(t)=p S(t-\tau) E(t-\tau) R(t-\tau)$ and duration of patient is infectious. Tian-Mu Chen, et.al [33] investigated the including effect of time delay to acquire the following nonlinear differential equations:

$$
\left\{\begin{array}{l}
\frac{d S(t)}{d t}=b+\gamma I(t)+\epsilon R(t)-(\mu+p) S(t)-\beta S(t) I(t), \\
\frac{d E(t)}{d t}=\beta S(t) I(t)-\mu E(t)-\eta E(t) R(t), \\
\frac{d I(t)}{d t}=(\eta+\sigma \beta) E(t)-(\alpha+\mu+\gamma) I(t), \\
\frac{d R(t)}{d t}=p S(t-\tau) E(t-\tau) R(t-\tau)-(\epsilon+\mu+\sigma \beta) R(t),
\end{array}\right.
$$

where $\epsilon$ is the latent period of human infection in population no longer infectious due to full recovered. The aim of the research work is to discuss on SEIR delay model in (2). If $\tau=0$, the equation (2) narrates the population inputs between size of population and number of initial infections. The COVID-19 basic reproduction number for the system (2) is defined by

$$
R_{0}=\frac{(b+\gamma I+\epsilon A) \beta(\eta+\sigma \beta)}{\mu(\mu+p)(\alpha+\mu+\gamma)} .
$$

We have likewise determined the basic reproduction number $R_{0}$ classical SIR model and we have seen that if $R_{0}<1$ disease doesn't proliferate into the population yet on the off chance that $R_{0}>1$ infection will spread among the population. We presented an isolated SIR model and SEIR model portraying disease movement under the presumption that all contaminated individuals are separated after the hatching time frame so that they can't taint others. Ailment movement in these models is controlled by the basic reproduction number $R_{0}$, which is diverse contrasted with that for the standard SIR model. In the event that $R_{0}>1$ (95\%, ranges 1.4 to 3.9$)$, at that point the quantity of inertness contaminated people exponentially develops. Be that as it may, if $R_{0}<1$, at that point the number of contaminated rots exponentially. This investigation of $R_{0}$ catches the course of COVID-19 flare-up and subsequently reveals insight into understanding the patterns of the flare-up and gives some preventive, measure not to spread COVID-19 malady ( $97 \%$, ranges 2.47 to 3.9 ). This portrays the normal number of recently contaminated cells produced from one tainted cell toward the start of the irresistible procedure. 


\section{Preliminaries}

Let $S(t)=C([-\tau, 0] ; \mathfrak{R})$ be the continuous norm function of Banach space mappings. The initial conditions for the model (2) are given as follows [35]:

$$
\left\{\begin{array}{l}
S(t) \geq 0, E(t) \geq 0, I(t) \geq 0, R(t) \geq 0, t \in[-\tau, 0] \\
S(0)>0, E(0)>0, I(0)>0, R(0)>0
\end{array}\right.
$$

Let $(S(t), E(t), R(t))$ be three main variables of the system with initial conditions and verify that there is a unique solution. The accompanying lemma is helpful for examining the positivity of the bounded solutions.

Lemma 2.1 In the system $(S(t), E(t), R(t))$ of (2) with initial conditions (3), we assume that limsupS $(t)_{t \rightarrow+\infty} \leq \frac{b+\gamma I+\epsilon R}{\mu+p}$.

Proof. If there is $t_{1}>0$ with the end goal that $S\left(t_{1}\right)>\frac{b+\gamma I+\epsilon R}{\mu+p}$ and $\dot{S}\left(t_{1}\right)>0$, then we have that

$\dot{S}\left(t_{1}\right)=b+\gamma I+\epsilon R-(\mu+p) S(t)-\beta S(t) I(t) \leq-\beta S(t) I(t) \leq 0$.

Hence we have utilized $S\left(t_{1}\right)>\frac{b+\gamma I+\epsilon R}{\mu+p}$. This is an inconsistency to $\dot{S}\left(t_{1}\right)>0$. Along these lines, the finish of Lemma 2.1 is verified.

Lemma 2.2 Let $(S(t), E(t), I(t), R(t))$ be the system (2) with initial conditions (3). At that point $(S(t), E(t), I(t))$ and $R(t)$ are certain and there exists a positive constant $\Gamma>0$, to such an extent that $S(t)<\Gamma, E(t)<\Gamma, I(t)<\Gamma$ and $R(t)<\Gamma$ at an adequately huge time $t$.

Proof. Consider the equation (2), we get

$\mathrm{S}(t)=S(0) e^{-\int_{0}^{t}((\mu+p)-\beta I(\epsilon)) d \epsilon}+\int_{0}^{t}(b+\gamma I+\epsilon R) e^{-\int_{\eta}^{t}(((\mu+p)-\beta I(\epsilon))) d \epsilon} d \eta$,

$E(t)=E(0) e^{-\int_{0}^{t}(\mu+\eta z(\epsilon)) d \epsilon}+\int_{0}^{t} \beta S(\eta) I(\eta) e^{-\int_{0}^{t}(\mu+\eta z(\epsilon)) d \epsilon} d \eta$,

$I(t)=I(0) e^{-(\mu+\alpha+\gamma) t}+\int_{0}^{t}\left(\eta+\frac{\beta \sigma}{N}\right) E(\eta) e^{-(\mu+\alpha+\gamma)(t-\eta)} d \eta$,

$\mathrm{R}(t)=R(0) e^{-(\sigma \beta+\epsilon+\mu) t}+\int_{0}^{t} p S(\eta-\tau) E(\eta-\tau) R(\eta-\tau) e^{(\sigma \beta+\epsilon+\mu) t} d \eta$.

It is anything but difficult to see that $S(t)$ is positive on the existence interval. At that point, we demonstrate that $E(t)$ is positive. Truth be told, let $t_{1}>0$ be the first run through to such an extent that $E\left(t_{1}\right)=0$. From equation (2), we get $I(t-1)=I(0) e^{-(\mu+\alpha+\gamma) t_{1}}+\int_{0}^{t_{1}}(\eta+\sigma \beta) E(\eta) e^{-(\alpha+\mu+\gamma)(t-\eta)} d \eta>0$.

Then again, from the second equation of (2), we have $\dot{E}\left(t_{1}\right)=\left(t_{1}\right) I\left(t_{1}\right)>0$. This implies $E(t)<0$ for $t \in\left(t_{1}-, t_{1}\right)$, where is a subjectively small positive constant, which prompts an inconsistency. It is follows that $E(t)>0$ and $I(t)>0$. By the comparative contention as the above mentioned, it is difficult to obtain that $R(t)$ is positive. Here, we discuss the contentions for extreme solution of (2).

Here $N(t)=S(t)+E(t)+\frac{\mu}{2(\eta+\sigma \beta)} I(t)+\frac{\eta(\mu+p)}{p(b+\gamma I+\epsilon R)} R(t+\tau)$,

and assume $q=\min \left\{(\mu+p), \frac{\mu}{2},(\alpha+\mu+\gamma),(\epsilon+\mu+\sigma \beta)\right\}$. From (2), we get

$$
\begin{aligned}
& \frac{d}{d t}[N(t)]=b+\gamma I+\epsilon R-(\mu+p) S(t)-\frac{\mu}{2} E(t)-\eta E(t) R(t)-\frac{\mu(\alpha+\mu+\gamma)}{2(\eta+\sigma \beta)} I(t)+\frac{\eta(\mu+p)}{b+\gamma I+\epsilon R} S(t) E(t) R(t) \\
& -\frac{\eta(\epsilon+\mu+\sigma \beta)(\mu+p)}{p(b+\gamma I+\epsilon R)} R(t+\tau) \\
& \leq(b+\gamma I+\epsilon R)-(\mu+p) S(t)-\frac{\mu}{2} E(t)-\frac{\mu(\alpha+\mu+\gamma)}{2(\eta+\sigma \beta)} I(t)-\frac{\eta(\epsilon+\mu+\sigma \beta)(\mu+p)}{p(b+\gamma I+\epsilon R)} R(t+\tau) \\
& <b+\gamma I+\epsilon R-q\left[S(t)+E(t)+\frac{\mu}{2(\eta+\sigma \beta)} I(t)+\frac{\eta(\mu+p)}{p(b+\gamma I+\epsilon R)} I(t+\tau)\right]=b+\gamma I+\epsilon R-q .
\end{aligned}
$$

Along these lines, $N(t)<\frac{b+\gamma I+\epsilon R}{(\mu+p) \eta}$ for all large t. Subsequently, $S(t), E(t), I(t)$ and $R(t)$ are at last limited by any positive constant $\Gamma$. Hence, finishes the verification of Lemma 2.2. 


\section{Theorems for Stability analysis}

There are three equilibria for system (2):

(i). COVID-19 Infection free equilibrium:

$E_{0}=\left(\frac{b+\gamma I+\epsilon R}{(\mu+p)}, 0,0,0\right)$.

(ii). COVID-19 infection absent equilibrium:

$E_{1}=\left(\frac{\mu(\alpha+\mu+\gamma)}{\beta}(\eta+\sigma \beta), \frac{(b+\gamma I+\epsilon R) \beta(\eta+\sigma \beta)-\mu(\mu+p)(\alpha+\mu+\gamma)}{\mu \beta(\eta+\sigma \beta)}, \frac{(b+\gamma I+\epsilon R) \beta(\eta+\sigma \beta)-\mu(\mu+p)(\alpha+\mu+\gamma)}{\mu \beta(\alpha+\mu+\gamma)}, 0\right)$.

(iii). COVID-19 infection present equilibrium:

$\bar{E}=\left(\bar{E}_{1}, \bar{E}_{2}, \bar{E}_{3}, \bar{E}_{4}\right)$,

where $\bar{E}_{1}=\frac{p(\alpha+\mu+\gamma)(b+\gamma I+\epsilon R)-\beta(\eta+\sigma \beta)(\epsilon+\mu+\sigma \beta)}{p(\mu+p)(\alpha+\mu+\gamma)}$,

$$
\begin{aligned}
& \bar{E}_{2}=\frac{(\epsilon+\mu+\sigma \beta)(\mu+p)(\alpha+\mu+\gamma)}{p(\alpha+\mu+\gamma)(b+\gamma I+\epsilon R)-\beta(\eta+\sigma \beta)(\epsilon+\mu+\sigma \beta)}, \\
& \bar{E}_{3}=\frac{(\eta+\sigma \beta)(\epsilon+\mu+\sigma \beta)(\mu+p)}{p(\alpha+\mu+\gamma)(b+\gamma I+\epsilon R)-\beta(\eta+\sigma \beta)(\epsilon+\mu+\sigma \beta)}, \\
& \bar{E}_{4}=\frac{1}{\eta}\left[\frac{\beta(\eta+\sigma \beta)(p(\alpha+\mu+\gamma)(b+\gamma I+\epsilon R)-\beta(\eta+\sigma \beta)(\epsilon+\mu+\sigma \beta))}{p(\mu+p)(\alpha+\mu+\gamma)^{2}}-\mu\right] .
\end{aligned}
$$

\subsection{Stability of COVID-19 Infection free equilibrium}

The nonlinear differential equation of (2) at the point $E_{0}$ is

$$
\left\{\begin{array}{l}
\frac{d S(t)}{d t}=-(\mu+p) S(t)-\frac{\beta(b+\gamma I+\epsilon R)}{(\mu+p)} I(t), \\
\frac{d E(t)}{d t}=-\mu E(t)+\frac{\beta(b+\gamma I+\epsilon R)}{(\mu+p)} I(t), \\
\frac{d I(t)}{d t}=(\eta+\sigma \beta) E(t)-(\alpha+\mu+\gamma) I(t), \\
\frac{d R(t)}{d t}=-(\epsilon+\mu+\sigma \beta) R(t) .
\end{array}\right.
$$

The polynomial equation for (4) is

$$
(\lambda+(\epsilon+\mu+\sigma \beta))(\lambda+(\mu+p))\left[\lambda^{2}+(\mu+(\alpha+\mu+\gamma)) \lambda+\mu(\alpha+\mu+\gamma)-\frac{(\eta+\sigma \beta) \beta(b+\gamma I+\epsilon R)}{(\mu+p)}\right]=0 .
$$

Two of the roots of the polynomial equation (5) is $\lambda_{1}=-(\epsilon+\mu+\sigma \beta), \lambda_{2}=-(\mu+p)$. The other roots are calculated by

$$
\lambda^{2}+(\mu+(\alpha+\mu+\gamma)) \lambda+\mu(\alpha+\mu+\gamma)-\frac{(\eta+\sigma \beta) \beta(b+\gamma I+\epsilon R)}{(\mu+p)}=0
$$

If $R_{0}<1$, then $\mu(\alpha+\mu+\gamma)-\frac{(\eta+\sigma \beta) \beta(b+\gamma I+\epsilon R)}{(\mu+p)}>0$, and $(\mu+(\alpha+\mu+\gamma))^{2}-4\left(\mu(\alpha+\mu+\gamma)-\frac{(\eta+\sigma \beta) \beta(b+\gamma I+\epsilon R)}{(\mu+p)}\right)>0$.

$$
\lambda_{3,4}=\frac{-(\mu+(\alpha+\mu+\gamma)) \pm \sqrt{(\mu+(\alpha+\mu+\gamma))^{2}-4\left(\mu(\alpha+\mu+\gamma)-\frac{(\eta+\sigma \beta) \beta(b+\gamma I+\epsilon R)}{(\mu+p)}\right)}}{2} .
$$

The equation (6) has negative real roots. It has the accompanying theorem. If $R_{0}<1$, then $E_{0}$ is locally asymptotic stable by developing a Lyapunov functional. If $R_{0}>1$, then $E_{0}$ is unstable.

Theorem 3.1 If $R_{0}<1$, then prove that $E_{0}$ is globally asymptotic stable.

Proof. For Lyapunov functional,

$V=\frac{1}{2}\left[S(t)-\frac{(\epsilon+\mu+\sigma \beta)+\gamma I+\epsilon R}{(\mu+\eta)}\right]^{2}+\frac{(\epsilon+\mu+\sigma \beta)+\gamma I+\epsilon R}{(\mu+\eta)} E(t)+m I(t)+\frac{\eta}{p} R(t)+\eta \int_{t-\tau}^{t} S(\theta) E(\theta) R(\theta) d \theta$,

where $m>0$. We have

$$
\begin{aligned}
& \left.V^{\prime}\right|_{(5)}=\left[S(t)-\frac{(b+\gamma I+\epsilon R)}{(\mu+p)}\right]\left[-(\mu+p)\left(S(t)-\frac{(b+\gamma I+\epsilon R)}{(\mu+p)}\right)-\beta S(t) I(t)\right]+\frac{(b+\gamma I+\epsilon R)}{(\mu+p)}[\beta S(t) I(t)-\mu E(t)-\eta E(t) R(t)] \\
& +\mathrm{m}[(\eta+\sigma \beta) E(t)-(\alpha+\mu+\gamma) I(t)]-\frac{\eta(\epsilon+\mu+\sigma \beta)}{p} R(t)+\eta S(t) E(t) R(t) .
\end{aligned}
$$


Since $\beta S(t) I(t)=\beta I(t)\left[S(t)-\frac{(b+\gamma I+\epsilon R)}{(\mu+p)}\right]+\frac{\beta(b+\gamma I+\epsilon R)}{(\mu+p)} I(t)$, we have

$$
\begin{aligned}
& \left.V^{\prime}\right|_{(5)}=-(\mu+p)\left[S(t)-\frac{(b+\gamma I+\epsilon R)}{(\mu+p)}\right]^{2}-\beta I(t)\left[S(t)-\frac{(b+\gamma I+\epsilon R)}{(\mu+p)}\right]^{2}+\eta S(t) R(t)\left[S(t)-\frac{(b+\gamma I+\epsilon R)}{(\mu+p)}\right] \\
& -\left(\frac{(b+\gamma I+\epsilon R) \mu}{(\mu+p)}-(\eta+\sigma \beta) m\right) E(t)-\left((\alpha+\mu+\gamma) m-\frac{\beta(b+\gamma I+\epsilon R)^{2}}{(\mu+p)^{2}}\right) I(t)-\frac{\eta(\epsilon+\mu+\sigma \beta)}{p} R(t) .
\end{aligned}
$$

Hence $R_{0}<1$ decreases to $\frac{(b+\gamma I+\epsilon R) \mu}{(\eta+\sigma \beta)(\mu+p)}-\frac{\beta(b+\gamma I+\epsilon R)^{2}}{(\alpha+\mu+\gamma)(\mu+p)^{2}}>0,\left(m \in\left[\frac{\beta(b+\gamma I+\epsilon R)^{2}}{(\alpha+\mu+\gamma)(\mu+p)^{2}}, \frac{(b+\gamma I+\epsilon R) \mu}{(\eta+\sigma \beta)(\mu+p)}\right]\right)$, such that $\frac{(b+\gamma I+\epsilon R) \mu}{(\mu+p)}-(\eta+\sigma \beta) m>0$ and $(\alpha+\mu+\gamma) m-\frac{\beta(b+\gamma I+\epsilon R)^{2}}{(\mu+p)^{2}}>0$.

Letting $S(t), E(t), R(t)$ are positive and $S(t) \leq \frac{(b+\gamma I+\epsilon R)}{(\mu+p)}$ holds, we have that $\left.V^{\prime}\right|_{(5)} \leq 0$, and $\left.V^{\prime}\right|_{(5)}=0$ iff $(S(t), E(t), I(t), R(t))=\left(\frac{(b+\gamma I+\epsilon R)}{(\mu+p)}, 0,0,0\right)$.

\subsection{Stability of COVID-19 infection absent equilibrium}

Letting $E_{1}=(\tilde{S}, \tilde{E}, \tilde{I}, 0)=\left(\frac{\mu(\alpha+\mu+\gamma)}{\beta(\eta+\sigma \beta)}, \frac{(b+\gamma I+\epsilon R) \beta-\mu(\mu+p)(\alpha+\mu+\gamma)}{\mu \beta(\alpha+\mu+\gamma)}, \frac{(b+\gamma I+\epsilon R) \beta(\eta+\sigma \beta)}{\mu \beta(\alpha+\mu+\gamma)}, 0\right)$, the linearized form of equations of system (2) at $E_{1}$ is

$$
\left\{\begin{array}{l}
\frac{d S(t)}{d t}=-((\mu+p)+\beta \tilde{I}) S(t)-\beta \tilde{S} I(t), \\
\frac{d E(t)}{d t}=\beta \tilde{I} S(t)-\mu E(t)+\beta \tilde{S} I(t)-\eta \tilde{E} R(t), \\
\frac{d I(t)}{d t}=(\eta+\sigma \beta) E(t)-(\alpha+\mu+\gamma) I(t), \\
\frac{d R(t)}{d t}=p \tilde{S} \tilde{E} R(t-\tau)-(\epsilon+\mu+\sigma \beta) R(t) .
\end{array}\right.
$$

The characteristic polynomial equation of (7) is

$\left(\lambda-p \tilde{S} \tilde{E} e^{-\lambda \tau}+(\epsilon+\mu+\sigma \beta)\right)\left(\lambda^{3}+a_{1} \lambda^{2}+a_{2} \lambda+a_{3}\right)=0$,

where

$a_{1}=\mu+(\alpha+\mu+\gamma)+(\mu+p)+\frac{(b+\gamma I+\epsilon R) \beta(\eta+\sigma \beta)-\mu(\mu+p)(\alpha+\mu+\gamma)}{\mu(\alpha+\mu+\gamma)}$,

$a_{2}=(\mu+(\alpha+\mu+\gamma))\left((\mu+p)+\frac{(b+\gamma I+\epsilon R) \beta(\eta+\sigma \beta)-\mu(\mu+p)(\alpha+\mu+\gamma)}{\mu(\alpha+\mu+\gamma)}\right)$,

$a_{3}=(b+\gamma I+\epsilon R) \beta(\eta+\sigma \beta)-\mu(\mu+p)(\alpha+\mu+\gamma)$.

First we obtain

$$
\lambda^{3}+a_{1}^{2}+a_{2} \lambda+a_{3}=0 .
$$

Clearly, if $R_{0}>1$, we have $a_{1}=\mu+(\alpha+\mu+\gamma)+(\mu+p)+\frac{(b+\gamma I+\epsilon R) \beta(\eta+\sigma \beta)-\mu(\mu+p)(\alpha+\mu+\gamma)}{\mu(\alpha+\mu+\gamma)}>0$ and

$$
\begin{aligned}
& a_{3}=(b+\gamma I+\epsilon R) \beta(\eta+\sigma \beta)-\mu(\mu+p)(\alpha+\mu+\gamma) . \\
& a_{1} a_{2}-a_{3}=\left(\mu+(\alpha+\mu+\gamma)+(\mu+p)+\frac{(b+\gamma I+\epsilon R) \beta(\eta+\sigma \beta)-\mu(\mu+p)(\alpha+\mu+\gamma)}{\mu(\alpha+\mu+\gamma)}\right)(\mu+(\alpha+\mu+\gamma)) \\
& \left((\mu+p)+\frac{(b+\gamma I+\epsilon R) \beta(\eta+\sigma \beta)-\mu(\mu+p)(\alpha+\mu+\gamma)}{\mu(\alpha+\mu+\gamma)}\right)-((b+\gamma I+\epsilon R) \beta(\eta+\sigma \beta)-\mu(\mu+p)(\alpha+\mu+\gamma)) \\
& =\mu^{2}(\mu+p)+\frac{\mu((b+\gamma I+\epsilon R) \beta(\eta+\sigma \beta)-\mu(\mu+p)(\alpha+\mu+\gamma))}{(\alpha+\mu+\gamma)}+\mu(\mu+p)(\alpha+\mu+\gamma) \\
& +\left((\alpha+\mu+\gamma)+(\mu+p)+\frac{(b+\gamma I+\epsilon R) \beta(\eta+\sigma \beta)-\mu(\mu+p)(\alpha+\mu+\gamma)}{\mu(\alpha+\mu+\gamma)}\right)
\end{aligned}
$$

$\left(\mu(\mu+p)+\frac{(b+\gamma I+\epsilon R) \beta(\eta+\sigma \beta)-\mu(\mu+p)(\alpha+\mu+\gamma)}{\mu(\alpha+\mu+\gamma)}+(\alpha+\mu+\gamma)(\mu+p)+\frac{(b+\gamma I+\epsilon R) \beta(\eta+\sigma \beta)-\mu(\mu+p)(\alpha+\mu+\gamma)}{\mu(\alpha+\mu+\gamma)}\right)>0$. By Routh-Hurwitz criteria, (8) has no positive roots. So, we investigate the another polynomial equation

$$
\lambda-p \tilde{S} \tilde{E} e^{-\lambda \tau}+(\epsilon+\mu+\sigma \beta)=0 .
$$

For $\tau=0, \lambda=\frac{\beta(\eta+\sigma \beta) p(\alpha+\mu+\gamma)(b+\gamma I+\epsilon R)-\beta^{2}(\eta+\sigma \beta)^{2}(\epsilon+\mu+\sigma \beta)-\mu p(\mu+p)(\alpha+\mu+\gamma)^{2}}{\beta^{2}(\eta+\sigma \beta)^{2}}$. Obviously, if $R_{0}<1+\frac{\beta^{2}(\eta+\sigma \beta)^{2}(\epsilon+\mu+\sigma \beta)}{\mu p(\mu+p)(\alpha+\mu+\gamma)^{2}}$, then $\phi<0$, which illustrations that the roots of (9) for some $\phi>0$ and $\tau>0$. From (9), we have

$$
\left\{\begin{array}{l}
\phi=-\frac{(\alpha+\mu+\gamma) p((b+\gamma I+\epsilon R) \beta(\eta+\sigma \beta)-\mu(\mu+p)(\alpha+\mu+\gamma))}{\beta^{2}(\eta+\sigma \beta)^{2}} \sin \phi \tau, \\
(\epsilon+\mu+\sigma \beta)=\frac{(\alpha+\mu+\gamma) p(s \beta(\eta+\sigma \beta)-\mu(\mu+p)(\alpha+\mu+\gamma))}{\beta^{2} k^{2}} \cos \phi \tau,
\end{array}\right.
$$


which implies that $\phi^{2}=p^{2}\left[\frac{(\alpha+\mu+\gamma) p((b+\gamma I+\epsilon R) \beta(\eta+\sigma \beta)-\mu(\mu+p)(\alpha+\mu+\gamma))}{\beta^{2}(\eta+\sigma \beta)^{2}}\right]^{2}-(\epsilon+\mu+\sigma \beta)^{2}$.

Note that if $1<R_{0}<1+\frac{\beta^{2}(\eta+\sigma \beta)^{2}(\epsilon+\mu+\sigma \beta)}{\mu p(\mu+p)(\alpha+\mu+\gamma)^{2}}$, then $\phi^{2}<0$. If $1<R_{0}<1+\frac{\beta^{2}(\eta+\sigma \beta)^{2}(\epsilon+\mu+\sigma \beta)}{\mu p(\mu+p)(\alpha+\mu+\gamma)^{2}}$, then the COVID-19 infection $E_{1}$ is locally asymptotic stable. If $1<R_{0}>1+\frac{\beta^{2}(\eta+\sigma \beta)^{2}(\epsilon+\mu+\sigma \beta)}{\mu p(\mu+p)(\alpha+\mu+\gamma)^{2}}$, then the COVID-19 infection $E_{1}$ is unstable.

\subsection{Stability of COVID-19 infection present equilibrium}

In COVID-19 infection, the effects of time delay $\tau$ is a bifurcation parameter and it goes through a stationary values. The COVID-19-present equilibrium occurs direct stability and Hopf bifurcation. As a matter of first importance, we interpret the equilibrium $\bar{E}=(\bar{S}, \bar{E}, \bar{I}, \bar{R})$ of system (2) to the source. Let $S_{1}(t)=S(t)-\bar{S}, E_{1}(t)=E(t)-\bar{E}, I_{1}(t)=$ $I(t)-\bar{I}, R_{1}(t)=R(t)-\bar{R}$. For effortlessness, we likewise use $S(t), E(t), I(t), R(t)$ rather than $S_{1}(t), E_{1}(t), I_{1}(t), R_{1}(t)$. The system (2) becomes

$$
\left\{\begin{array}{l}
\frac{d S(t)}{d t}=-((\mu+p)+\beta \bar{I}) S(t)-\beta S(t) I(t)-\beta \bar{S} I(t), \\
\frac{d E(t)}{d t}=\beta S(t) I(t)+\beta \bar{I} S(t)-(\mu+\eta \bar{R}) E(t)+\beta \bar{S} I(t)-\eta \bar{E} R(t)-\eta E(t) R(t), \\
\frac{d I(t)}{d t}=(\eta+\sigma \beta) E(t)-(\alpha+\mu+\gamma) I(t), \\
\frac{d R(t)}{d t}=[p S(t-\tau) E(t-\tau) R(t-\tau)+p \bar{E} S(t-\tau) R(t-\tau)+p \bar{S} E(t-\tau) R(t-\tau)+p \bar{S} \bar{E} R(t-\tau) \\
+p \bar{R} S(t-\tau) E(t-\tau)+p \bar{E} \bar{R} S(t-\tau)+p \bar{S} \bar{R} E(t-\tau)-(\epsilon+\mu+\sigma \beta) R(t) .
\end{array}\right.
$$

Then, the origin $(0,0,0,0)^{T}$ is steady state of (11) and the linearized system of equation (11) at the origin is

$$
\left\{\begin{array}{l}
\frac{d S(t)}{d t}=-((\mu+p)+\beta \bar{I}) S(t)-\beta S(t) I(t)-\beta \bar{S} I(t) \\
\frac{d E(t)}{d t}=\beta S(t) I(t)+\beta \bar{I} S(t)-(\mu+\eta \bar{R}) E(t)+\beta \bar{S} I(t)-\eta \bar{E} R(t)-\eta E(t) R(t), \\
\frac{d(t)}{d t}=(\eta+\sigma \beta) E(t)-(\alpha+\mu+\gamma) I(t) \\
\frac{d R(t)}{d t}=p S(t-\tau) E(t-\tau) R(t-\tau)+p \bar{E} S(t-\tau) R(t-\tau)+p \bar{S} E(t-\tau) R(t-\tau)+p \bar{S} \bar{E} R(t-\tau) \\
+p \bar{R} S(t-\tau) E(t-\tau)+p \bar{E} \bar{R} S(t-\tau)+p \bar{S} \bar{R} E(t-\tau)-(\epsilon+\mu+\sigma \beta) R(t) .
\end{array}\right.
$$

The trivial solution of equation (12) is asymptotic stable and the equation (11) is locally asymptotic stable. The strength of the polynomial equation (12) is given by

$$
\Omega(\lambda)=\lambda^{4}+x_{1} \lambda^{3}+x_{2} \lambda^{2}+x_{3} \lambda+x_{4}-\left(x_{5} \lambda^{3}+x_{6} \lambda^{2}+x_{7} \lambda+x_{8}\right) e^{-\lambda \tau}
$$

where

$x_{1}=(\epsilon+\mu+\sigma \beta)+(\mu+p)+\beta \bar{I}+(\alpha+\mu+\gamma)+\mu+\eta \bar{R}$,

$x_{2}=(\epsilon+\mu+\sigma \beta)(\mu+p)+(\epsilon+\mu+\sigma \beta) \beta \bar{I}+(\epsilon+\mu+\sigma \beta)(\alpha+\mu+\gamma)+(\mu+p)(\alpha+\mu+\gamma)+\beta \bar{I}(\alpha+\mu+\gamma)+(\mu+$ $\eta \bar{R})((\epsilon+\mu+\sigma \beta)+(\mu+p)+\beta \bar{I})$,

$x_{3}=(\epsilon+\mu+\sigma \beta)(\mu+p)(\alpha+\mu+\gamma)+(\epsilon+\mu+\sigma \beta) \beta \bar{I}(\alpha+\mu+\gamma)+(\mu+\eta \bar{R})((\epsilon+\mu+\sigma \beta)(\mu+p)+(\epsilon+\mu+\sigma \beta) \beta \bar{I})+$ $(\eta+\sigma \beta) \beta^{2} \bar{S} \bar{I}$,

$x_{4}=(\epsilon+\mu+\sigma \beta)(\eta+\sigma \beta) \beta^{2} \bar{S} \bar{I}$,

$x_{5}=(\epsilon+\mu+\sigma \beta)$,

$x_{6}=(\epsilon+\mu+\sigma \beta)(\mu+p)+(\epsilon+\mu+\sigma \beta) \beta \bar{I}+(\epsilon+\mu+\sigma \beta)(\alpha+\mu+\gamma)+\mu(\epsilon+\mu+\sigma \beta)$,

$x_{7}=(\epsilon+\mu+\sigma \beta)(\mu+p)(\alpha+\mu+\gamma)+(\epsilon+\mu+\sigma \beta) \beta \bar{I}(\alpha+\mu+\gamma)+(\mu+\eta \bar{R})((\epsilon+\mu+\sigma \beta)(\mu+p)+(\epsilon+\mu+\sigma \beta) \beta \bar{I})-$ $(\epsilon+\mu+\sigma \beta) \eta \bar{R}((\mu+p)+\beta \bar{I}+(\alpha+\mu+\gamma))$,

$x_{8}=(\epsilon+\mu+\sigma \beta)(\eta+\sigma \beta) \beta^{2} \bar{S} \bar{I}-(\epsilon+\mu+\sigma \beta)(\mu+p)(\alpha+\mu+\gamma) \eta \bar{R}$.

Theorem 3.2 If the solution of (12) is locally asymptotic stable, then $\tau=0$ and $R_{0}>1+\frac{\beta^{2}(\eta+\sigma \beta)^{2}(\epsilon+\mu+\sigma \beta)}{\mu p(\mu+p)(\alpha+\mu+\gamma)^{2}}$. Proof. Let $\tau=0$. From (13),

$$
\lambda^{4}+\left(x_{1}+x_{5}\right) \lambda^{3}+\left(x_{2}-x_{6}\right) \lambda^{2}+\left(x_{3}-x_{7}\right) \lambda+x_{4}-x_{8}=0 .
$$

Since $R_{0}>1+\frac{\beta^{2}(\eta+\sigma \beta)^{2}(\epsilon+\mu+\sigma \beta)}{\mu p(\mu+p)(\alpha+\mu+\gamma)^{2}}, \bar{S}>0, \bar{E}>0, \bar{I}>0, \bar{R}>0$. 
By the method of Routh-Hurwitz criteria, we get

$x_{9}=x_{1}-x_{5}=\beta \bar{I}+\eta \bar{R}+3 \mu+p+\alpha+\gamma>0$,

$x_{10}=\left(x_{1}-x_{5}\right)\left(x_{2}-x_{6}\right)-\left(x_{3}-x_{7}\right)$

$=((\mu+p)+\beta \bar{I}+(\alpha+\mu+\gamma)+\mu+\eta \bar{R}[(\mu+p)(\alpha+\mu+\gamma)+\beta \bar{I}(\alpha+\mu+\gamma)+(\mu+\eta \bar{R})((\epsilon+\mu+\sigma \beta)+(\mu+p)+\beta \bar{I})-\mu(\epsilon+\mu+\sigma \beta)]-$ $(\epsilon+\mu+\sigma \beta)(\eta+\sigma \beta) \beta^{2} \bar{S} \bar{I}-(\epsilon+\mu+\sigma \beta) \eta \bar{R}((\mu+p)+\beta \bar{I}+(\alpha+\mu+\gamma)) \times((\mu+p)+\beta \bar{I}+(\alpha+\mu+\gamma))[(\mu+p)(\alpha+\mu+$ $\gamma)+(\alpha+\mu+\gamma) \beta \bar{I}+(\mu+\eta \bar{R})((\mu+p)+\eta \bar{R})((\mu+p)+\beta \bar{I})]+[(\mu+p)(\alpha+\mu+\gamma)+(\mu+\eta \bar{R})((\mu+\eta)+\beta \bar{I})+(\epsilon+\mu+\sigma \beta) \eta \bar{R}]$ $x_{11}=\left|\begin{array}{ccc}x_{1}-x_{5} & x_{3}-x_{7} & 0 \\ 1 & x_{2}-x_{6} & x_{4}-x_{8} \\ 0 & x_{1}-x_{5} & x_{3}-x_{7}\end{array}\right|=\left(x_{1}-x_{5}\right)\left[\left(x_{2}-x_{6}\right)\left(x_{3}-x_{7}\right)-\left(x_{1}-x_{5}\right)\left(x_{4}-x_{8}\right)\right]\left(x_{3}-x_{7}\right)^{2}$.

Let $m=\mu+\eta \bar{R}, n=(\mu+p)+\beta \bar{I}$. Thus,

$x_{11}=[(\alpha+\mu+\gamma) m(n-(\mu+p))+\eta(\epsilon+\mu+\sigma \beta) \bar{R} n+\eta(\epsilon+\mu+\sigma \beta) \bar{R}(\alpha+\mu+\gamma)]\left((\alpha+\mu+\gamma) n^{2}+m n^{2}+(\alpha+\mu+\right.$ $\left.\gamma)^{2} n+(\alpha+\mu+\gamma) m n+(\mu+p)(\alpha+\mu+\gamma) m+m^{2} n+(\epsilon+\mu+\sigma \beta) m \eta \bar{R}\right)$

$-(\epsilon+\mu+\sigma \beta)(\mu+p)(\alpha+\mu+\gamma) \eta \bar{R}\left(n^{2}+(\alpha+\mu+\gamma)^{2}+2(\alpha+\mu+\gamma) n+m^{2}+2 m n+2 m(\alpha+\mu+\gamma)\right)$

$=(\alpha+\mu+\gamma) m(n-(\mu+p))\left((\alpha+\mu+\gamma) n^{2}+m n^{2}+(\alpha+\mu+\gamma)^{2} n+(\alpha+\mu+\gamma) m n+(\mu+p)(\alpha+\mu+\gamma) m+m^{2} n+(\epsilon+\mu+\sigma \beta) m \eta \bar{R}\right)+$ $(\epsilon+\mu+\sigma \beta) \eta \bar{R}(\alpha+\mu+\gamma)^{2} m(n-(\mu+p))+(\epsilon+\mu+\sigma \beta)(\alpha+\mu+\gamma)^{3} \eta \bar{R}(n-d)+2(\epsilon+\mu+\sigma \beta) \eta \bar{R}(\alpha+\mu+\gamma)^{2} n(n-(\mu+p))+$ $(\epsilon+\mu+\sigma \beta) \eta \bar{R}(\alpha+\mu+\gamma) m^{2}(n-(\mu+p))+2(\epsilon+\mu+\sigma \beta) \eta \bar{R}(\alpha+\mu+\gamma) m n(n-(\mu+p))+(\epsilon+\mu+\sigma \beta) \eta \bar{R}(\alpha+\mu+\gamma)^{2} m(n-$ $(\mu+p))+(\epsilon+\mu+\sigma \beta) \eta \bar{R} m n^{3}+(\epsilon+\mu+\sigma \beta) \eta \bar{R}(\mu+p)(\alpha+\mu+\gamma) m n+(\epsilon+\mu+\sigma \beta) \eta \bar{R} m^{2} n^{2}+(\epsilon+\mu+\sigma \beta)^{2} m \eta^{2} \bar{R}^{2} n+$ $(\epsilon+\mu+\sigma \beta)^{2} \eta^{2} \bar{R}^{2}(\alpha+\mu+\gamma) m$.

We have $x_{11}>0$, since $n-(\mu+p)>0$.

$\mathrm{x}_{12}=\left|\begin{array}{cccc}x_{1}-x_{5} & x_{3}-x_{7} & 0 & 0 \\ 1 & x_{2}-x_{6} & x_{4}-x_{8} & 0 \\ 0 & x_{1}-x_{5} & x_{3}-x_{7} & 0 \\ 0 & 1 & x_{2}-x_{6} & x_{4}-x_{8}\end{array}\right|=\mathrm{a}_{4} x_{11}$.

Taking note of that $a_{4}=(\epsilon+\mu+\sigma \beta)(\mu+p)(\alpha+\mu+\gamma) \eta \bar{x}$, it is anything but difficult to acquire that $x_{12}>0$. Subsequently, the real parts are negative in (14). This completes the verification of the Theorem 3.2. Here the roots of $\Omega(\lambda)=0$ have negative real roots. Hence, there exists a $\tau_{0}>0$ to such that $\tau \in\left[0, \tau_{0}\right)$ in (13), we have

$$
\Omega(\lambda)=0, \operatorname{Re}(\lambda)<0 \text { for } \tau \in\left[0, \tau_{0}\right)
$$

what's more, when $\tau=\tau_{0}, \operatorname{Re}(\lambda)<0$. To decide this $\tau_{0}$ and the related simply $\phi_{0} i\left(\phi_{0}>0\right)$ imaginary roots, we understand (13) with $\lambda=\phi_{0} i$. For straight forwardness, we use $\tau, \phi$ rather than $\tau_{0}, \phi_{0}$. From (13), we have

$$
\begin{gathered}
\phi^{4}-x_{1} \phi^{3} i-x_{2} \phi^{2}+x_{3} \phi i+x_{4}-\left(-x_{5} \phi^{3} i-x_{6} \phi^{2}+x_{7}+x_{8}\right) \\
(\cos \phi \tau-i \sin \phi \tau)=0 .
\end{gathered}
$$

Comparing the coefficients of real and imaginary parts, we get

$$
\left\{\begin{array}{l}
\left(x_{8}-x_{6} \phi^{2}\right) \cos \phi \tau+\left(x_{7} \phi-x_{5} \phi^{3}\right) \sin \phi \tau=\phi^{4}-x_{2} \phi^{2}+x_{4} \\
\left(x_{5} \phi^{3}-x_{7} \phi\right) \cos \phi \tau+\left(x_{8}-x_{6} \phi^{2}\right) \sin \phi \tau=x_{1} \phi^{3}-x_{3} \phi
\end{array}\right.
$$

$$
\begin{aligned}
& \quad \cos \phi \tau=\frac{1}{\Delta}\left|\begin{array}{cc}
\phi^{4}-x_{2} \phi^{2}+x_{4} & x_{7} \phi-x_{5} \phi^{3} \\
x_{1} \phi^{3}-x_{3} \phi & x_{8}-x_{6} \phi^{2}
\end{array}\right| \\
& =\frac{1}{\Delta}\left[\left(\mathrm{x}_{1} x_{5}-x_{6}\right) \phi^{6}+\left(x_{8}+x_{2} x_{6}-x_{1} x_{7}-x_{3} x_{5}\right) \phi^{4}+\left(x_{3} x_{7}-x_{2} x_{8}-x_{4} x_{6}\right) \phi^{2}+x_{4} x_{8}\right] \\
& =\frac{1}{\Delta}\left(c_{1} \phi^{6}+c_{2} \phi_{4}+c_{3} \phi^{2}+c_{4}\right) . \\
& \sin \phi \tau=\frac{1}{\Delta}\left|\begin{array}{cc}
x_{8}-x_{6} \phi^{2} & \phi^{4}-x_{2} \phi^{2}+x_{4} \\
x_{5} \phi^{3}-x_{7} \phi & x_{1} \phi^{3}-x_{3} \phi
\end{array}\right|
\end{aligned}
$$$$
=\left(-\frac{\phi}{\Delta}\right)\left(x_{5} \phi^{6}+\left(x_{1} x_{6}-x_{7}-x_{2} x_{5}\right) \phi^{4}+\left(x_{2} x_{7}+x_{4} x_{5}-x_{3} x_{6}-x_{1} x_{8}\right) \phi^{2}+\left(x_{3} x_{8}-x_{4} x_{7}\right)\right)
$$$$
=-\frac{\phi}{\Delta}\left(x_{9} \phi^{6}+x_{10} \phi^{4}+x_{11} \phi^{2}+x_{12}\right)
$$

where $\Delta=\left|\begin{array}{cc}x_{8}-x_{6} \phi^{2} & x_{7} \phi-x_{5} \phi^{3} \\ x_{4} \phi^{3}-x_{7} \phi & x_{8}-x_{6} \phi^{2}\end{array}\right|$ 
$=\left(x_{8}-x_{6} \phi^{2}\right)^{2}+\left(x_{7}-x_{5} \phi^{3}\right)^{2}=x_{5} \phi^{6}+\left(x_{6}-2 x_{5} x_{7}\right) \phi^{4}+\left(x_{7}^{2}-2 x_{6} x_{8}\right) \phi^{2}+x_{8}^{2}=\left(e_{1} \phi^{6}+e_{2} \phi^{4}+e_{3} \phi^{2}+e_{4}\right)>0$.

Here $\sin ^{2} \phi \tau+\cos ^{2} \phi \tau=1$, it follows that

$$
\phi^{14}+x_{13} \phi^{12}+x_{14} \phi^{10}+x_{15} \phi^{8}+x_{16} \phi^{6}+x_{17} \phi^{4}+x_{18} \phi^{2}+x_{19}=0
$$

where

$$
\begin{aligned}
& x_{13}=\frac{1}{x_{9}^{2}}\left(c_{1}^{2}+2 x_{9} x_{10}-e_{1}^{2}\right), \\
& x_{14}=\frac{1}{x_{9}^{2}}\left(2 c_{1} c_{2}+x_{10}^{2}+2 x_{9} x_{11}-2 e_{1} e_{3}\right), \\
& x_{15}=\frac{1}{x_{9}^{2}}\left(c_{2}^{2}+2 c_{1} c_{3}+2 x_{9} x_{12}+2 x_{10} x_{11}-e_{2}^{2}-2 e_{1} e_{3}\right), \\
& x_{16}=\frac{1}{x_{9}^{2}}\left(2 c_{1} c_{4}+2 c_{2} c_{3}+x_{11}^{2}+2 x_{10} x_{12}-2 e_{1} e_{4}-2 e_{2} e_{3}\right), \\
& x_{17}=\frac{1}{x_{9}^{2}}\left(c_{3}^{2}+2 c_{2} c_{4}+2 x_{11} x_{12}-e_{3}^{2}-2 e_{2} e_{4}\right), \\
& x_{18}=\frac{1}{x_{9}^{2}}\left(2 c_{3} c_{4}+x_{12}^{2}-2 e_{3} e_{4}\right), \\
& x_{19}=\frac{1}{x_{9}^{2}}\left(c_{4}^{2}-e_{4}^{2}\right) .
\end{aligned}
$$

Denoting : $x=\phi^{2}$, (18) becomes

$$
x^{7}+x_{13} x^{6}+x_{14} x^{5}+x_{15} x^{4}+x_{16} x^{3}+x_{17} x^{2}+x_{18} x+x_{19}=0
$$

First, $x=0$ is not a root of (19) if $x_{8} \neq 0$. There is no positive real root in (19). Therefore $\phi=\sqrt{x}$ does not get the solution. Hence bifurcation parameter $\tau$ does not occur and Hopf bifurcation is not evaluate. The equation (19) always has positive real roots. Let the hypothesis as follows:

$\left(\Omega_{1}\right)$ : Equation (19) is possible one positive real root;

$\left(\Omega_{2}\right): \Lambda \triangleq\left[4 \phi^{6}+3\left(x_{1}^{2}-2 x_{2}-x_{5}^{2}\right) \phi^{4}+2\left(x_{2}^{2}-x_{6}^{2}+2 x_{4}+x_{5} x_{7}-2 x_{1} x_{3}\right) \phi^{2}+x_{3}^{2}-x_{7}^{2}+2 x_{6} x_{8}-2 x_{2} x_{4}\right]>0$ for any $\phi>0$. Let $x_{0}$ be the positive roots of (19), denoting $\phi_{0}=\sqrt{x_{0}}$. From the above, we get

$\tau_{j}=\frac{1}{\phi_{0}}\left(\cos ^{-1}\left(\frac{c_{1} \phi_{0}^{6}+c_{2} \phi_{0}^{4}+c_{3} \phi_{0}^{2}+c_{4}}{e_{1} \phi_{0}^{6}+e_{2} \phi_{0}^{4}+e_{3} \phi_{0}^{2}+e_{4}}\right)+2 j \pi\right), \mathrm{j}=0,1,2,3, \ldots$

$\tau_{0}=\frac{1}{\phi_{0}} \cos ^{-1}\left(\frac{c_{1} \phi_{0}^{6}+c_{2} \phi_{0}^{4}+c_{3} \phi_{0}^{2}+c_{4}}{e_{1} \phi_{0}^{6}+e_{2} \phi_{0}^{4}+e_{3} \phi_{0}^{2}+e_{4}}\right), j=0$.

The set of ordered pair is $\left(\phi_{0}, \tau_{0}\right)$ to find the polynomial roots of (13) neighborhood $\tau_{0}$ and differentiating with respect to $\tau$, we get

$$
\left[\frac{d \lambda}{d \tau}\right]^{-1}=\frac{-\left(4 \lambda^{3}+3 x_{1} \lambda^{2}+2 x_{2} \lambda+x_{3}\right) e^{\lambda} \tau}{\lambda\left(x_{5} \lambda^{3}+x_{6} \lambda^{2}+x_{7} \lambda+x_{8}\right)}+\frac{3 x_{5} \lambda^{2}+2 x_{6} \lambda+x_{7}}{\lambda\left(x_{5} \lambda^{3}+x_{7} \lambda^{2}+x_{7} \lambda+x_{8}\right)}-\frac{\tau}{\lambda}
$$

Letting (20), we have

$$
\begin{gathered}
\operatorname{Re}\left[\frac{d \lambda}{d \tau}\right]^{-1}=\frac{1}{\phi \nabla}\left\{\left(3 x_{1} \phi^{2}-x_{3}\right)\left[\left(x_{5} \phi^{3}-x_{7} \phi\right) \cos \phi \tau+\left(x_{8}-x_{8} \phi^{2}\right) \sin \phi \tau\right]\right. \\
+\left(4 \phi^{3}-2 x_{2} \phi\right)\left[\left(x_{8}-x_{6} \phi^{2}\right) \cos \phi \tau-\left(x_{5} \phi^{3}-x_{7} \phi\right) \sin \phi \tau\right] \\
+\left(x_{7}-3 x_{5} \phi^{2}\right)\left(x_{5} \phi^{3}-x_{7} \phi\right)+2 x_{6} \phi\left(x_{8}-x_{6} \phi^{2}\right) \\
=\frac{1}{\nabla}\left[4 \phi^{6}+3\left(x_{2}^{2}-2 x_{2}-x_{5}^{2}\right) \phi^{4}+2\left(x_{2}^{2}-x_{6}^{2}+2 x_{4}+2 x_{5} x_{7}-2 x_{1} x_{3}\right) \phi^{2}\right. \\
\left.+x_{3}^{2}-x_{7}^{2}+2 x_{6} x_{8}-2 x_{2} x_{4}\right],
\end{gathered}
$$

where, $\nabla=\left(x_{5} \phi^{3}-x_{7} \phi\right)^{2}+\left(x_{8}-x_{6} \phi^{2}\right)^{2}>0$. If $\left(\Omega_{2}\right)$ is satisfied, then $(20)>0$ will hold for any $\phi>0$.

So, $\operatorname{sign}\left\{\operatorname{Re}\left[\frac{d \lambda}{d \tau}\right] \mid \tau=\tau_{0}\right\}=\operatorname{sign}\left\{\operatorname{Re}\left[\frac{d \lambda}{d \tau}\right] \mid \tau=\tau_{0}\right\} \stackrel{\Delta}{=} \operatorname{sign}()=$.1 .

Therefore, the roots of (14) have negative real parts. If $\tau=\tau_{0}$, then other negative real roots have in $\Omega(\lambda)=0$. In $(14)$, if $\tau \in\left[0, \tau_{0}\right)$ and $\left(\Omega_{1}, \Omega_{2}\right)$ assumption, then the COVID-19 infection is stable. Similarly, if $\tau>\tau_{0}$, then the COVID-19 infection is unstable and equation (2) undertakes bifurcation at $\tau=\tau_{0}$. 


\section{Numerical Experiment}

Let us consider the parameters $b=0.5, \gamma=0.008, \epsilon=0.1, \mu=0.0018, p=0.5, \beta=0.1923, \eta=0.1, \sigma=0.5, \alpha=$ 0.5 with $(S(0), E(0), I(0), R(0))$ [34]. In the event that (19) has no positive roots, at that point the COVID-19 infection present equilibrium is locally asymptotic stable. On the off chance that $R_{0}=2.47$, at that point COVID-19 disease present equilibrium $E=(3.1,1.4,10.01,2.01)$. From (19), we have that

$$
x^{7}+500.01 x^{6}+10025 x^{5}+23423 x^{4}+4099 x^{3}+28.1 x^{2}+0.1 x+6.110^{-5}=0,
$$

has real negative roots. Therefore the equilibria is locally asymptotic stable and it represents Hopf bifurcation. Obviously, $R_{0}=1.92$, and the COVID-19 infection present equilibrium is $E=(3.01,1.3,8.1,3.3)$. From (19), we have that

$$
x^{7}+477.2304 x^{6}+47123 x^{5}+33257 x^{4}+5008 x^{3}+27.1 x^{2}-1.3 x-0.001=0
$$

have a positive real roots and others have negative real roots. Accordingly, $\phi_{0}=\sqrt{x}=0.1$ It isn't hard to evaluate the bifurcation stationary value is $\tau_{0}=1.96$. Also, it is anything but difficult to prove that $\Lambda=2.8>0$, i.e., $\left(\Omega_{2}\right)$ is fulfilled. The phase diagrams of the system (2) is asymptotic stable when $\tau=0.9<\tau_{0}$ (see Figure 1). Also, the phase diagrams of the system (2) undergoes Hopf bifurcation when $\tau=2>\tau_{0}$ (see Figure 2). We utilize the serious cases and deaths in the individual response work, rather than deaths as it were. We additionally increment the power of the legislative activity to such an extent that the model results to a great extent coordinate the watched, with a revealing proportion. To be specific just an extent of the model created cases will be accounted for as a general rule. Consequently it would be testing given a generally brief time frame arrangement, and a few other obscure parameters to be assessed.

\section{Conclusion}

There is a shortage of epidemiological information about the rising coronavirus, which would be of essential significance to structure and execute auspicious, specially appointed viable general well being intercessions, isolate and travel limitations. We have contemplated a general SEIR model of COVID-19 infection with delay. If $R_{0}<1$, then stability of the disease-free equilibrium derived by Lyapunov techniques. Furthermore the effects of time delay $\tau=0$, the COVID-19 infection is either absent or present equilibrium when $R_{0}>1$. Here $1<R_{0}<1+\frac{\beta^{2}(\eta+\sigma \beta)^{2}(\epsilon+\mu+\sigma \beta)}{\mu p(\mu+p)(\alpha+\mu+\gamma)^{2}}$, then $E_{1}$ is stable. If $R_{0}>1+\frac{\beta^{2}(\eta+\sigma \beta)^{2}(\epsilon+\mu+\sigma \beta)}{\mu p(\mu+p)(\alpha+\mu+\gamma)^{2}}$, then $E_{1}$ is unstable. Hence, $\tau>0,1<R_{0}<1+\frac{\beta^{2}(\eta+\sigma \beta)^{2}(\epsilon+\mu+\sigma \beta)}{\mu p(\mu+p)(\alpha+\mu+\gamma)^{2}}, E_{1}$ is stable. The basic reproductive ratio $R_{0}>1+\frac{\beta^{2}(\eta+\sigma \beta)^{2}(\epsilon+\mu+\sigma \beta)}{\mu p(\mu+p)(\alpha+\mu+\gamma)^{2}}$, if the susceptible cells birth rate is high. Therefore the linearized system of (2) has no real positive roots and the stability is stable. The polynomial equation (19) has a single real positive root when $\tau<\tau_{0}$. The COVID-19 infection present equilibrium is stable. If $\tau>\tau_{0}$, the equilibrium solutions are unstable and a Hopf bifurcation occurs. Suppose, the equation (19) has more than one positive roots, it does not exit. In future, The further investigation is needed to this system. The controlling of reproduction number ratios proposes that the outbreak might be more genuine than what has been accounted for up until now, given the specific period of expanding social contacts, justifying powerful, severe general well being measures planned to relieve the weight produced by the spreading of the new infection.

\section{Availability of data and material}

To this article have no data sets where generated or analyzed in the current study Data.

\section{Competing Interests}

The authors declare there are no competing interests.

\section{Funding}

We are gratefully acknowledged the SRM Institute of Science and Technology, Chennai, India, for financial assistance in the form of a Research fellowship (URF). 


\section{Authors Contributions}

For the writing of this paper all authors are equally contributed and also read and agreed the final copy of the manuscript.

\section{Acknowledgments}

We would like to thank Dr. Vetrivel V, Professor, Department of Mathematics, Indian Institute of Technology Madras, Chennai, for their enriching suggestions and ideas.

\section{References}

[1] World Health Organization. Novel Coronavirus Japan (ex-China). World Health Organization. cited April 16, 2020. Available: https://www.who.int/ csr/don/16-April-2020-novel-coronavirus-japan-ex-china/en/.

[2] Zhou P, Yang XL, Wang XG, Hu B, Zhang L, Zhang W, et al. A pneumonia outbreak associated with a new coronavirus of probable bat origin. Nature. 2020. https://doi.org/10.1038/s41586-020-2012-7.

[3] Li Q, Guan X, Wu P, Wang X, Zhou L, Tong Y, et al. Early transmission dynamics in Wuhan, China, of novel coronavirus-infected pneumonia. N Engl J Med. 2020. https://doi.org/10.1056/NEJMoa2001316.

[4] Huang C, Wang Y, Li X, Ren L, Zhao J, Hu Y, et al. Clinical features of patients infected with 2019 novel coronavirus in Wuhan, China. Lancet. 2020. https://doi.org/10.1016/S0140-6736(20)30183-5.

[5] Zhu N, Zhang D, Wang W, Li X, Yang B, Song J, et al. A novel coronavirus from patients with pneumonia in China, 2019. N Engl J Med. 2020. https:// doi.org/10.1056/NEJMoa2001017.

[6] Zhao S, Musa SS, Lin Q, Ran J, Yang G, Wang W, et al. Estimating the unreported number of novel coronavirus (2019-nCoV) cases in China in the first half of January 2020: a data-driven Modelling analysis of the early outbreak. J Clin Med. 2020. https://doi.org/10.3390/jcm9020388.

[7] Dye C, Gay N. Epidemiology. Modeling the SARS epidemic. Science. 2003; 300:18845.

[8] Zhou G, Yan G. Severe acute respiratory syndrome epidemic in Asia. Emerg Infect Dis. 2003;9:160810.

[9] Wu Z, McGoogan JM. Characteristics of and important lessons from the Coronavirus disease 2019 (COVID-19) outbreak in China: summary of a report of 72,314 cases from the Chinese center for disease control and prevention. JAMA 2020; doi: http://dx.doi.org/10.1001/jama.2020.2648.

[10] Huang C, Wang Y, Li X, Ren L, Zhao J, Hu Y, et al. Clinical features of patients infected with 2019 novel coronavirus in Wuhan, China. Lancet 2020;

[11] Guan W, Ni Z, Hu Y, Liang W, Ou C, He J, et al. Clinical characteristics of 2019 novel coronavirus infection in China. NEJM 2020;, doi:http://dx.doi.org/10.1056/ NEJMoa2002032.

[12] Nishiura H, Kobayashi T, Yang Y, Hayashi K, Miyama T, Kinoshita R, et al. The rate of under ascertainment of novel Coronavirus (2019-nCoV) infection: estimation using Japanese passengers data on evacuation flights. J Clin Med 2020a;9(2) Available from: https://www.mdpi.com/2077-0383/9/2/419.

[13] Bogoch II, Watts A, Thomas-Bachli A, Huber C, Kraemer MUG, Khan K. Pneumonia of unknown etiology in Wuhan, China: potential for international spread via commercial air travel. J Travel Med 2020;, doi:http://dx.doi.org/10.1056/ NEJMoa2002032.

[14] Wu JT, Leung K, Leung GM. Now casting and forecasting the potential domestic and international spread of the 2019-nCoV outbreak originating in Wuhan, China: a modelling study. Lancet 2020; Available from: http://www.sciencedirect.com/science/article/pii/S1201971220300539.

[15] Qianying Lina, Shi Zhaob, Daozhou Gaod, Yijun Loue, Shu Yangf, Salihu S. Musae, Maggie H. Wangb, Yongli Caig, Weiming Wangg, Lin Yangh, Daihai Hee, A conceptual model for the coronavirus disease 2019 (COVID-19) outbreak in Wuhan, China with individual reaction and governmental action, International Journal of Infectious Diseases 93 (2020) 211216.

[16] Bauch, C. T. et al. (2005) Dynamically modeling SARS and other newly emerging respiratory illnesses: past, present, and future, Epidemiology, 16(6), pp. 791801

[17] Cauchemez, S. et al. (2016) Unraveling the drivers of MERS-CoV transmission, Proceedings of the National Academy of Sciences of the United States of America, 113(32), pp. 90819086

[18] Chan, J. F.-W. et al. (2020) A familial cluster of pneumonia associated with the 2019 novel coronavirus indicating person-to-person transmission: a study of a family cluster, The Lancet. doi: 10.1016/s0140-6736(20)30154-9.

[19] Chan, J. F.-W. et al. (2020) A familial cluster of pneumonia associated with the 2019 novel coronavirus indicating person-to-person transmission: a study of a family cluster, The Lancet. doi: 10.1016/s0140-6736(20)30154-9.

[20] Chowell, G. et al. (2004) Model parameters and outbreak control for SARS, Emerging infectious diseases, 10(7), pp. 12581263.

[21] Lessler, J. et al. (2009) Incubation periods of acute respiratory viral infections: a systematic review, The Lancet infectious diseases, 9(5), pp. 291300.

[22] Lipsitch, M. et al. (2003) Transmission dynamics and control of severe acute respiratory syndrome, Science, 300(5627), pp. 19661970.

[23] Tan, W. et al. (2020) A Novel Coronavirus Genome Identified in a Cluster of Pneumonia Cases Wuhan, China 20192020, China CDC Weekly. China CDC Weekly, 2(4), pp. 6162.

[24] Chen, Y.; Liu, Q.; Guo, D. Coronaviruses: Genome structure, replication, and pathogenesis. J. Med. Virol.2020.

[25] Hui, D.S.C.; Zumla, A. Severe acute respiratory syndrome: Historical, epidemiologic, and clinical features. Infect. Dis. Clin. North Am. $2019,33,869889$

[26] Killerby, M.E.; Biggs, H.M.; Midgley, C.M.; Gerber, S.I.; Watson, J.T. Middle East respiratory syndrome coronavirus transmission. Emerg. Infect. Dis. 2020, 26, 191198 . 
[27] Willman, M.; Kobasa, D.; Kindrachuk, J. A Comparative analysis of factors influencing two outbreaks of middle eastern respiratory syndrome (MERS) in Saudi Arabia and South Korea. Viruses 2019, 11, 1119.

[28] Cohen, J.; Normile, D. New SARS-like virus in China triggers alarm. Science 2020, 367, 234235.

[29] Lu, H.; Stratton, C.W.; Tang, Y.W. Outbreak of pneumonia of unknown etiology inWuhan China: The mystery and the miracle. J. Med. Virol. 2020.

[30] Rothe, C.; Schunk, M.; Sothmann, P.; Bretzel, G.; Froeschl, G.;Wallrauch, C.; Zimmer, T.; Thiel, V.; Janke, C.; Guggemos, W.; et al. Transmission of 2019-nCoV infection from an asymptomatic contact in Germany.N. Engl. J. Med. 2020.

[31] Hui, D.S.; Azhar, E.E.I.; Madani, T.A.; Ntoumi, F.; Kock, R.; Dar, O.; Ippolito, G.; Mchugh, T.D.; Memish, Z.A.; Drosten, C.; et al. The continuing 2019-nCoV epidemic threat of novel coronaviruses to global health-The latest 2019 novel coronavirus outbreak in Wuhan, China. Int. J. Infect. Dis. 2020, 91, 264266.

[32] Cheng, V.C.C.; Wong, S.C.; To, K.K.W.; Ho, P.L.; Yuen, K.Y. Preparedness and proactive infection control measures against the emerging Wuhan coronavirus pneumonia in China. J. Hosp. Infect. 2020.

[33] Tian-Mu Chen, Jia Rui, Qiu-Peng Wang, Ze-Yu Zhao, Jing-An Cui and Ling Yin, A mathematical model for simulating the phase-based transmissibility of a novel coronavirus, Infectious Diseases of Poverty, (2020) 9:24 https://doi.org/10.1186/s40249-020-00640-3.

[34] Zhao S, Lin Q, Ran J, Musa SS, Yang G, Wang W, et al. Preliminary estimation of the basic reproduction number of novel coronavirus (2019-nCoV) in China, from 2019 to 2020: a data-driven analysis in the early phase of the outbreak. Int J Infect Dis. 2020. https://doi.org/10.1016/j.ijid.2020.01.050.

[35] Chen T, Rui J, Wang Q, Zhao Z, Cui J-A, Yin L. A mathematical model for simulating the transmission of Wuhan novel Coronavirus. bioRxiv. 2020: 2020.2001.2019.911669. 

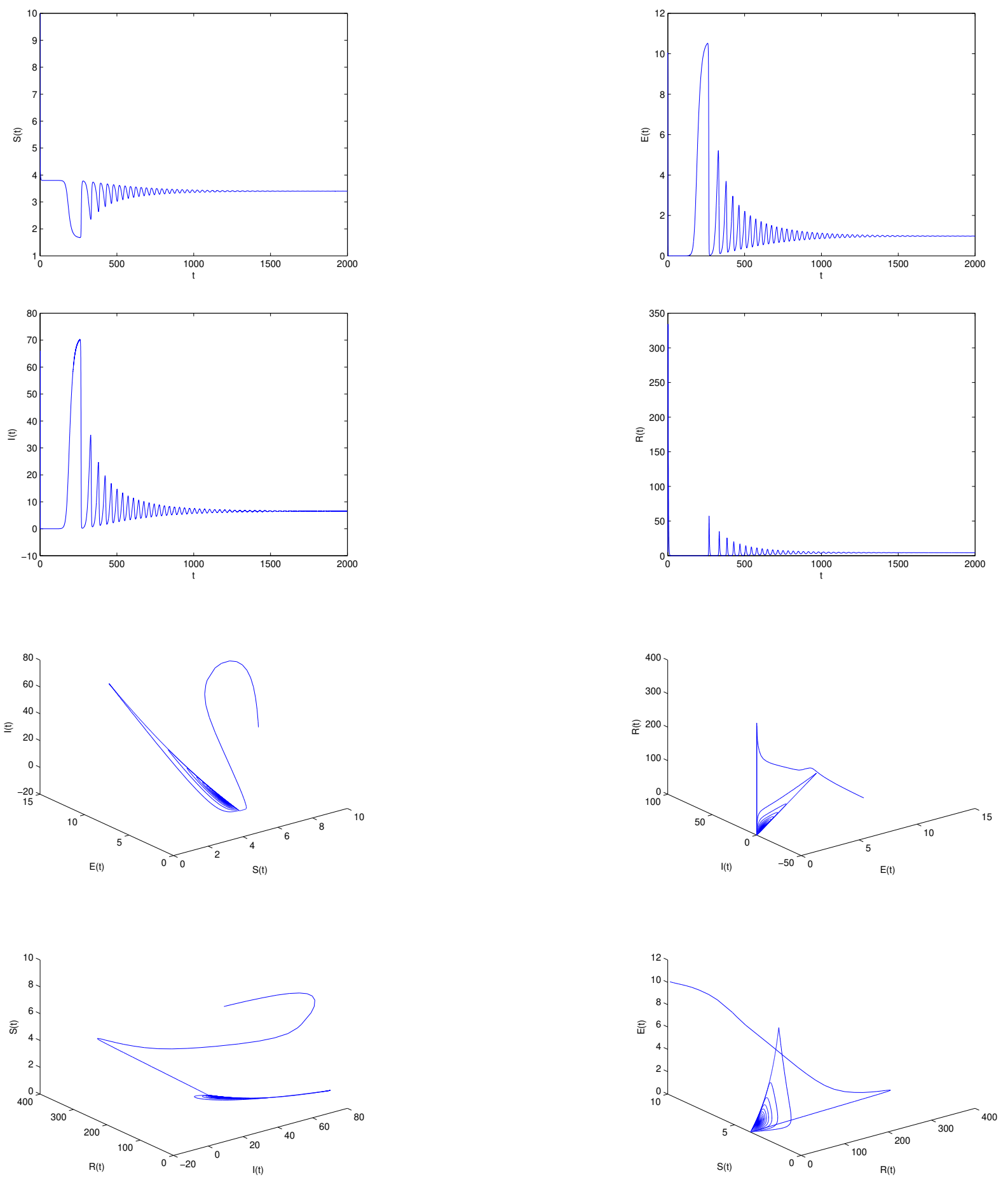

Figure 1: The phase diagrams of the system (2) is asymptotically stable when $\tau=0.9$ 

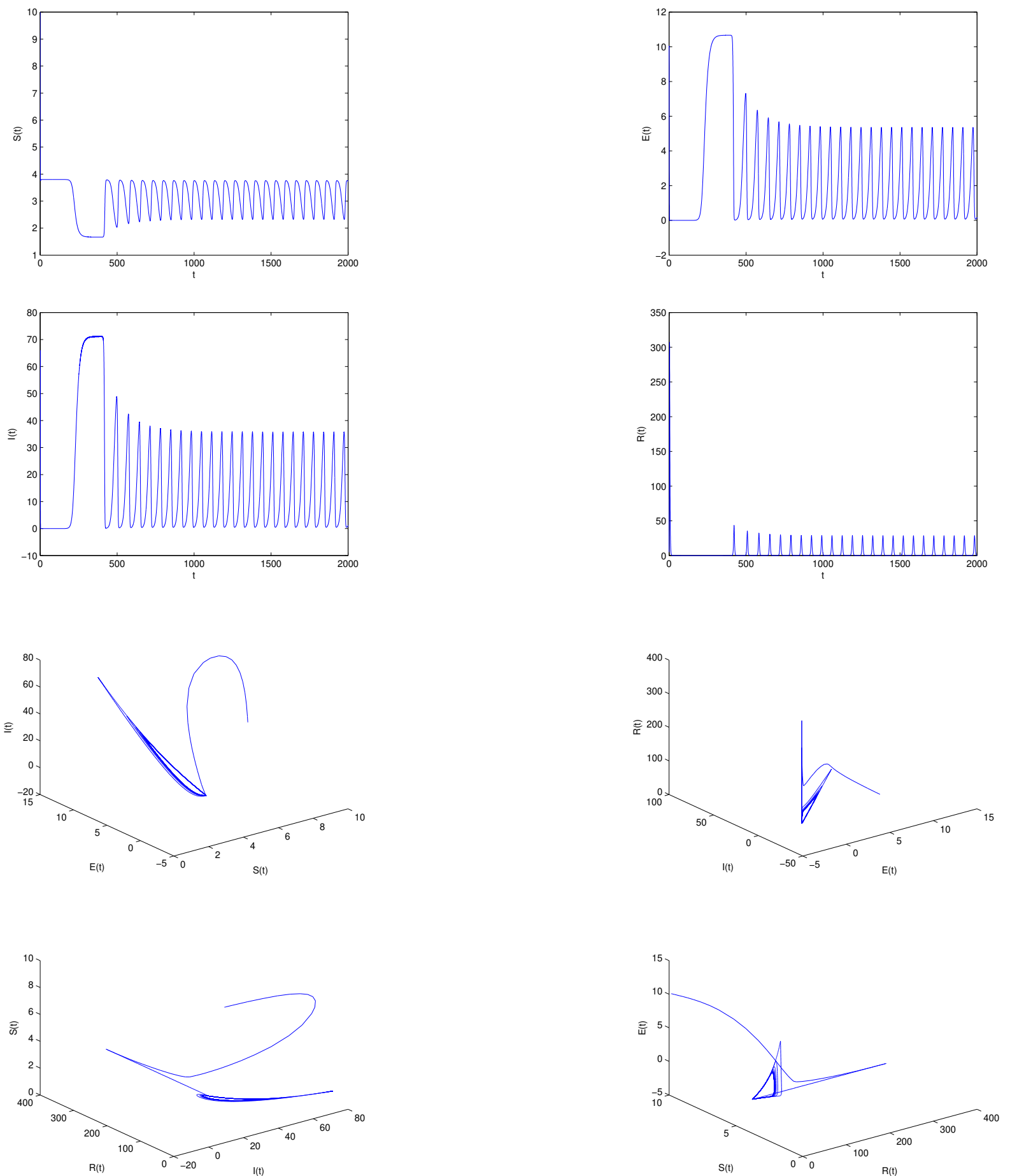

Figure 2: The phase diagrams of the system (2) undergoes Hopf bifurcation when $\tau=2$ 
Figures
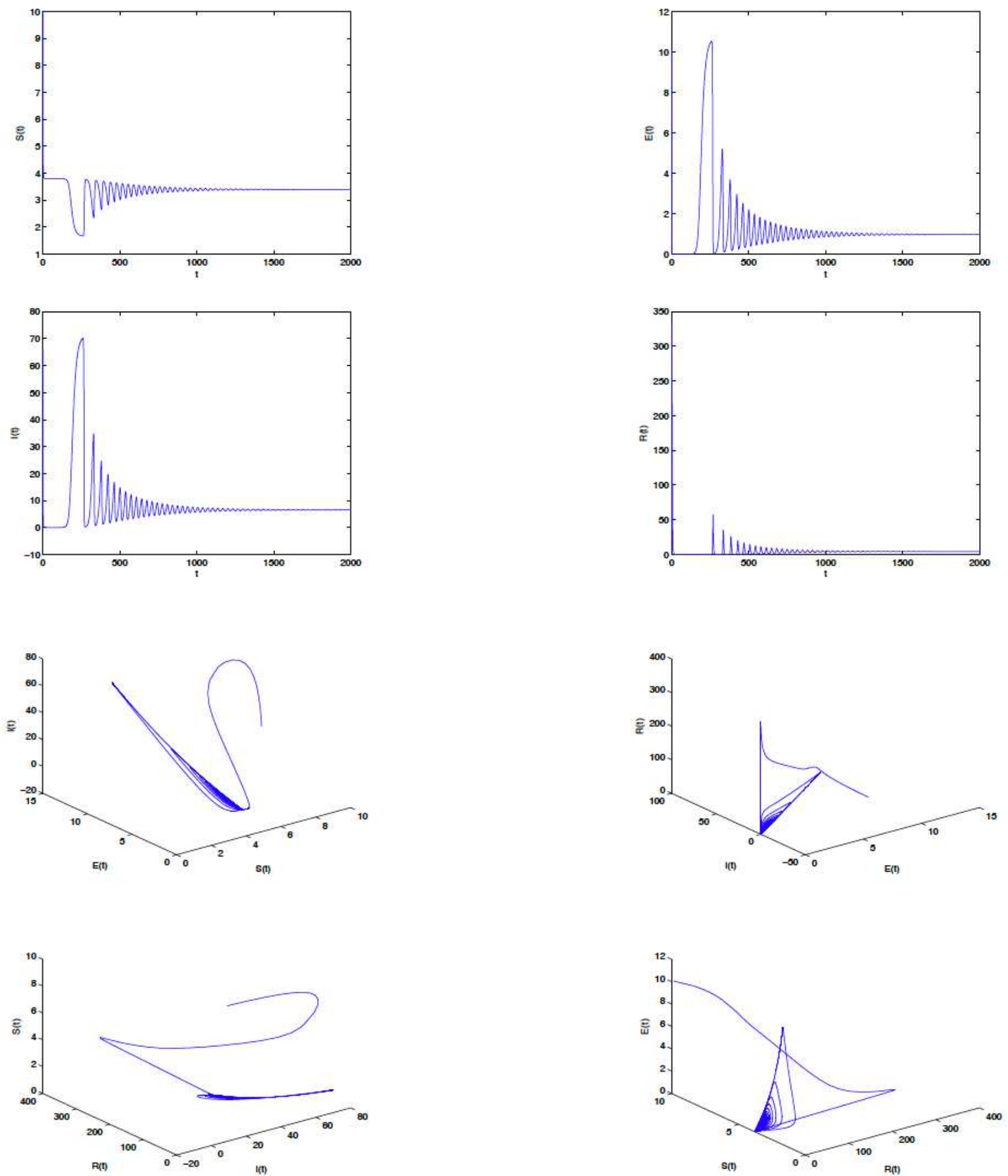

Figure 1

The phase diagrams of the system (2) is asymptotically stable when $\tau=0.9$ 

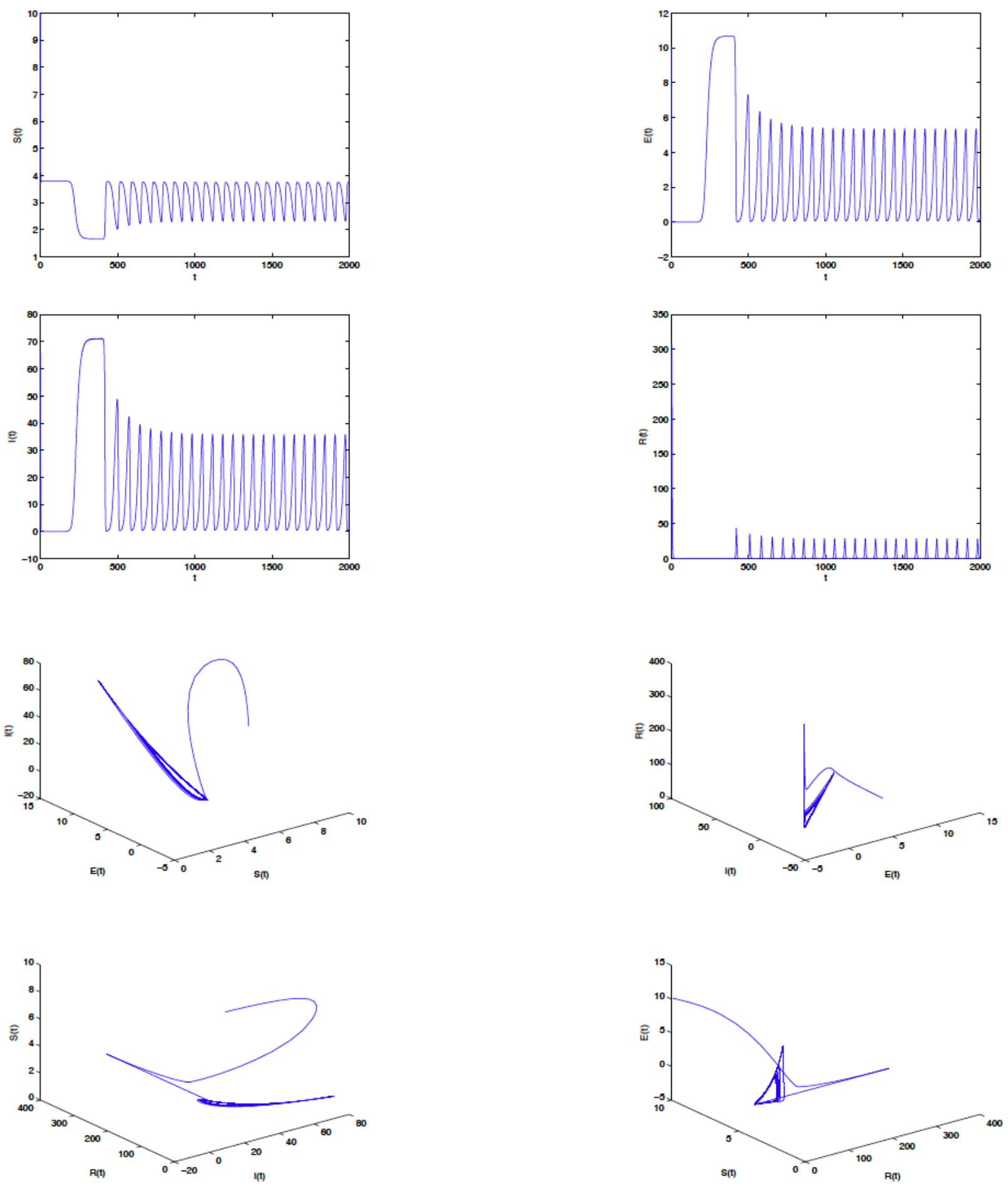

Figure 2

The phase diagrams of the system (2) undergoes Hopf bifurcation when $\tau=2$ 